\title{
Singly and doubly resonant femtosecond optical parametric oscillators for precision spectroscopy from the near-to mid-infrared
}

D. Reid, R. McCracken, Y. S. Cheng, O. Kara, J. Charsley, et al.

D. T. Reid, R. A. McCracken, Y. S. Cheng, O. Kara, J. M. Charsley, L. Maidment, "Singly and doubly resonant femtosecond optical parametric oscillators for precision spectroscopy from the near-to mid-infrared," Proc. SPIE 10638, Ultrafast Bandgap Photonics III, 106381F (8 May 2018); doi: 10.1117/12.2305133 


\title{
Singly-and doubly-resonant femtosecond optical parametric oscillators for precision spectroscopy from the near-to-mid-infrared
}

\author{
D. T. Reid ${ }^{* a}$, R. A. McCracken ${ }^{\mathrm{a}}$, Y. S. Cheng ${ }^{\mathrm{a}}$, O. Kara ${ }^{\mathrm{a}}$, J. M. Charsley and L. Maidment ${ }^{\mathrm{b}}$ \\ ${ }^{a}$ Scottish Universities Physics Alliance (SUPA), Institute of Photonics and Quantum Sciences, \\ School of Engineering and Physical Sciences, Heriot-Watt University, Edinburgh EH14 4AS, UK; \\ ${ }^{\mathrm{b}}$ Chromacity Ltd., Livingstone House, 43C Discovery Terrace, Riccarton EH14 4AP.
}

\begin{abstract}
Optical parametric oscillators (OPOs), pumped by Ti:sapphire and Yb-doped femtosecond lasers, provide unique capabilities to address a broad range of parameters of interest to precision spectroscopy. We review here a variety of OPOs under development that offer tuning from 1.5 to $13 \mu \mathrm{m}$, repetition rates from $100 \mathrm{MHz}$ to $10 \mathrm{GHz}$ and pulse durations from $<25$ fs to a few picoseconds. Spectroscopic techniques revealing the individual frequency comb modes are discussed, along with dual-comb spectroscopy at $3 \mu \mathrm{m}$ and from $6-8 \mu \mathrm{m}$.
\end{abstract}

Keywords: Optical parametric oscillator; spectroscopy; chemical sensing; femtosecond; infrared.

\section{INTRODUCTION}

Soon after their first realization in 1965 [1], the potential of optical parametric oscillators (OPOs) for spectroscopy was demonstrated experimentally. Femtosecond OPOs [2] were originally developed with the application of time-resolved spectroscopy in mind, where the spectral resolution is traded against temporal resolution in measurements such as the thermalization of hot carriers in semiconductors [3]. Only more recently has their potential for precision spectral measurements - previously the exclusive domain of narrow linewidth continuous-wave (cw) or nanosecond sourcesbeen fully understood and realized. This development has been progressed in two ways. The first exploits the broadband nature of femtosecond pulses to conduct Fourier-transform spectroscopy using a conventional Fourier transform spectrometer (FTS), often in the infrared (FTIR spectroscopy) where ro-vibrational features are present in the absorption spectra of molecules. In 2004 we reported [4] the first example of using a mid-infrared femtosecond OPO to conduct ro-vibrational spectroscopy of methane over a $14 \mathrm{THz}$ bandwidth, sufficient to span the $\mathrm{P}$, Q and R branches in the 3.3- $\mu \mathrm{m}\left(3000-\mathrm{cm}^{-1}\right)$ wavelength region. This approach transfers the burden of spectral resolution and calibration from the laser source to the spectrometer and has been applied in a variety of ways, including achieving certain embodiments of stand-off chemical spectroscopy. An extension of this approach is dual-comb spectroscopy (DCS) [5], which exploits the regularly spaced longitudinal mode structure of a femtosecond source to implement a form of Fouriertransform spectroscopy with no moving parts. Here, two femtosecond sources, which are identical in all respects except for a small difference in their repetition frequencies, are interfered to produce a radio-frequency (RF) comb that is a replica of the optical comb. When the light from one or both combs has sampled molecular absorption lines, the absorption spectrum is encoded on the RF comb spectrum and can be used to perform ultra-high precision spectroscopy revealing molecular line shapes and spectra. In 2013 we reported the first mid-infrared dual-comb spectroscopy using two MgO:PPLN optical parametric oscillators [6], carrying out spectroscopy of a methane:air mixture at $3.3 \mu \mathrm{m}$. This has recently been extended to longer wavelengths corresponding to the molecular fingerprint region by using an OPGaP OPO operating between 6 and $8 \mu \mathrm{m}$ [7]. The resolution of the spectral measurement in DCS is generally limited to the mode spacing of the combs, often $100 \mathrm{MHz}$ or higher, but the individual comb modes generated by a femtosecond OPO also have the potential to be used for spectroscopy, since their modal linewidths can be less than $100 \mathrm{kHz}$, and extend across a wide spectral range, making it possible to perform heterodyne measurements revealing the emission linewidth of an independent $\mathrm{cw}$ source [8].

The spectroscopic drivers for using femtosecond OPOs are usually the need to reach a specific wavelength region that is otherwise hard to access using lasers, or to address a particularly wide range of wavelengths either simultaneously or nearly simultaneously (with appropriate tuning), which again can be impossible to obtain from laser transitions. We will introduce two specific use-cases, one from astronomy, the other from stand-off chemical detection of hazardous agents.

Ultrafast Bandgap Photonics III, edited by Michael K. Rafailov, Proc. of SPIE Vol. 10638, $106381 \mathrm{~F} \cdot$ C $2018 \mathrm{SPIE} \cdot$ CCC code: $0277-786 \mathrm{X} / 18 / \$ 18 \cdot$ doi: $10.1117 / 12.2305133$ 
In the first example, there is a need for broadband spectral calibration of astronomical spectrographs, an application in which for some time laser frequency combs have been recognized as being a promising solution $[9,10]$. Currently the most demanding performance is required by the Extremely Large Telescope (ELT), whose 'HIRES' spectrograph targets a spectral bandwidth from $370-2400 \mathrm{~nm}$, and requires a laser comb that can address a similar range, with essentially gapfree coverage and mode spacings from a few $\mathrm{GHz}$ to around $30 \mathrm{GHz}$ [11]. This range is problematic to address from any single laser source, but the comb-preserving conversion from short to long wavelengths enabled by doubly-resonant OPOs makes them excellent candidates for such an instrument.

Stand-off chemical spectroscopy for purpose of the remote classification and identification of potentially hazardous agents requires broad spectral coverage, ideally in the spectroscopic fingerprint band extending from $6-20 \mu \mathrm{m}$. In this scenario the spatial coherence of an OPO gives it an immediate advantage over infrared thermal sources by allowing the beam to be directed over a long distance [12], while the spectral coverage can be orders of magnitude greater than that provided by sources based on laser gain media $[13,14]$.

The remainder of this paper describes in detail selected OPO configurations that are being developed for spectroscopic applications in astronomy and stand-off chemical sensing, outlining their designs and giving example of the performance which can be achieved.

\section{TI:SAPPHIRE-PUMPED OPO FREQUENCY COMBS FOR ASTRONOMY}

\subsection{Motivation}

The concept of utilising a frequency comb, with its extremely-high precision and stability, for use as a calibrator for astronomical spectrographs was immediately recognized as a promising technique for further increasing precision in extreme precision radial velocity (RV) measurements on its inception in 2007 [15]. The frequency-comb spectrograph calibrator ('astrocomb') has the potential to enable precision and accuracy enough to explore some truly novel science cases, to help answer profound questions such as 'Are the fundamental constants time-varying?'. The near-immediate recognition of the astronomy community is supported by the proof-of-concept experiment carried out by Steinmetz et al. within a year of the former's publication [9]. To date, the predominant resident calibration sources on high-resolution astronomical spectrographs are emission lamps, typically hollow-cathode Thorium-Argon (Th-Ar) lamps, or absorption cells, typically iodine reference cells. These calibration sources produce spectral lines associated with well-mapped atomic transitions and have contributed to the discovery of over 700 exoplanets through the radial velocity method alone [16]. The motivation for a new calibration source arises partly out of necessity, as the manufacturing of Th-Ar lamps with metallic cathodes, the type of Th-Ar lamp best suited for spectrograph calibration, has ceased worldwide [17], and in part due to scientific motivation from the astronomy community for RV precision on the $\sim 1 \mathrm{~cm} \mathrm{~s}^{-1} \mathrm{scale}$. Atomictransition-based sources have spectral lines sparsely positioned across the coverage of a spectrograph, and are entirely absent in the near-IR. Accurate modelling of the instrument profile with these calibration has seen their maximum RV precision limits realized at the $\sim 1 \mathrm{~m} \mathrm{~s}^{-1}$ level $[18,19]$.

\subsection{Performance requirements}

The application of high-resolution spectroscopy to characterize the orbits of Earth-like exoplanets in orbit around distant stars is determined by the magnitude of the radial velocity change effected on the star during the planet's orbit. Large planets in orbit close to their host star (so called "hot Jupiters") can cause radial velocity changes of $50 \mathrm{~m} \mathrm{~s}^{-1}$ [20], but Earth-like planets orbiting stars similar to our Sun can expect to cause a much smaller change, requiring a spectroscopic wavelength accuracy down to $10 \mathrm{~cm} \mathrm{~s}^{-1}$ [11] and precision of $1 \mathrm{~cm} \mathrm{~s}^{-1}$. The spectrometers used for this purpose have resolving powers of $100,000(\lambda / \Delta \lambda)$ or more, and utilize a cross-dispersed echelle-grating configuration. Comb-light with modal linewidths much narrower than the spectrometer resolution are introduced into the spectrometer, acting as a regularly spaced series of delta functions in frequency whose spacing can be used to obtain the wavelength solution (the mapping of pixel to wavelength) for the spectrometer. The wavelength dependence of the frequency resolution, $|\Delta f|=$ $c \Delta \lambda / \lambda^{2}$, means that the comb mode spacing must be increased for shorter wavelengths. Taking the E-ELT calibration requirements as an example, a spacing of $7 \mathrm{GHz}$ is sufficient in the infrared from $1155-2000 \mathrm{~nm}$, while a spacing of $20 \mathrm{GHz}$ is needed in the visible from $375-435 \mathrm{~nm}$ [21]. An example of the deployment of a practical astrocomb on the Southern African Large Telescope was recently reported [22]. 


\subsection{Architecture of a broadband astrocomb}

Simultaneously providing the required mode spacing and bandwidth directly from any femtosecond source is extremely challenging, so the prevailing approach is to start from a low-repetition-rate source and use Fabry-Pérot filtering to produce from this one or more combs of the required spacing. The architecture being progressed for the calibration of the ELT is shown in Fig. 1 [21] and is based on a 1-GHz Ti:sapphire laser that is frequency down-converted using a degenerate doubly-resonant OPO. Both the Ti:sapphire and OPO are spectrally broadened in supercontinuum fiber before a series of Fabry-Pérot etalons performs the necessary mode filtering, with shorter wavelengths being filtered to wider spacings than longer wavelengths.

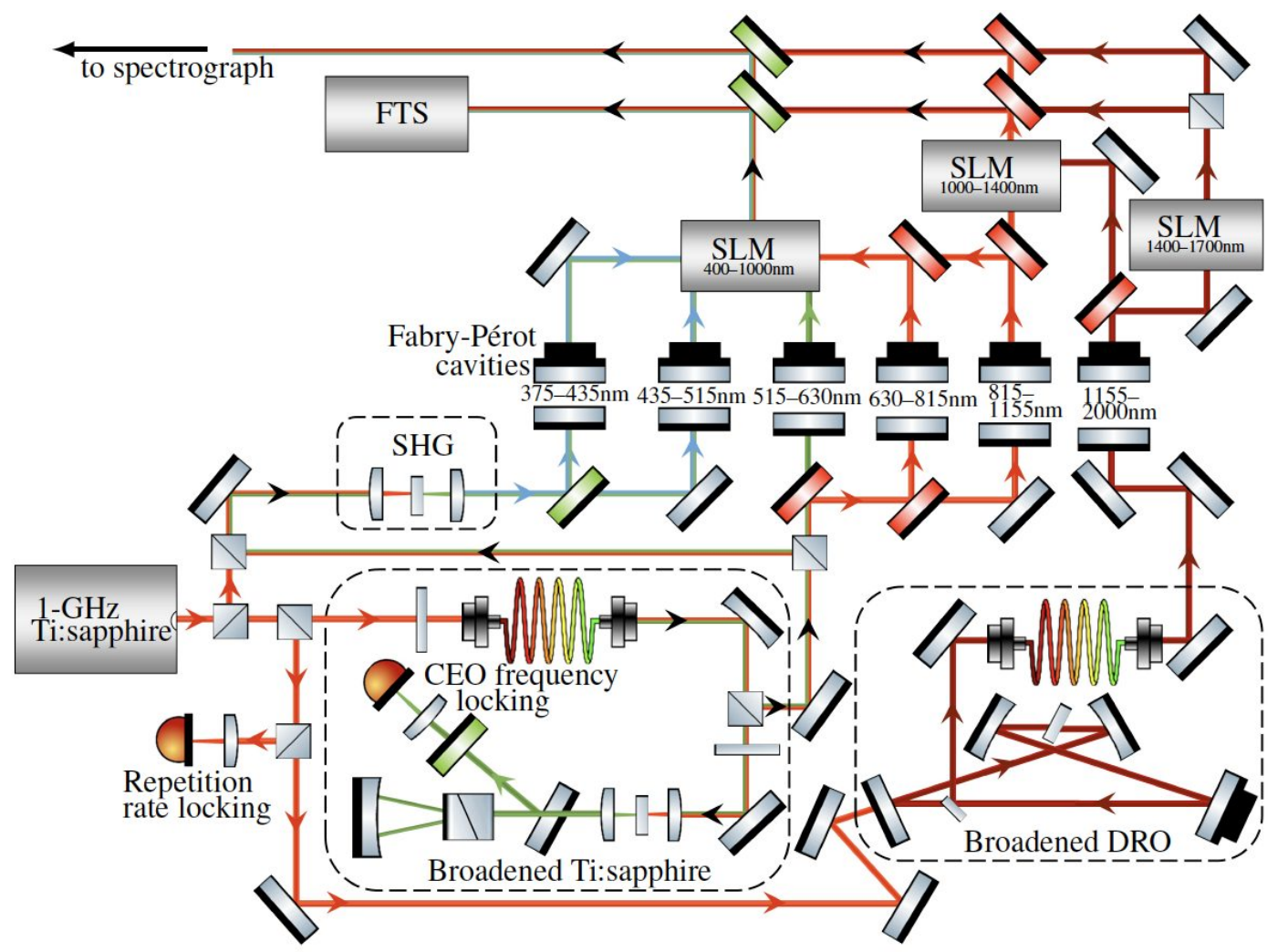

Fig. 1. Proposed architecture of the OPO-based laser-frequency-comb calibrator for HIRES.

\subsection{Ti:sapphire-pumped doubly-resonant degenerate 1-GHz OPO}

The 1-GHz OPO comb is based on a system describe previously [23] and is shown in Fig. 2. The pump source was a 1GHz Ti:sapphire laser (Gigajet, Laser Quantum) which produced 31-fs pulses at $803 \mathrm{~nm}$. The OPO was synchronously pumped by the Ti:sapphire laser, such that their cavity lengths $(\sim 300 \mathrm{~mm})$ were equal. The OPO was a 4-mirror ring cavity based around a $0.5-\mathrm{mm}$ length of PPKTP with a domain period of $26.5 \mu \mathrm{m}$ (Raicol Crystals) cut at Brewster's angle for $1.6 \mu \mathrm{m}$. PPKTP offers a number of practical advantages over MgO:PPLN for use in a Ti:sapphire pumped degenerate OPO, including lower material dispersion and broader phasematching bandwidth. Pump light was coupled into the cavity through mirror M1. A pair of curved mirrors with 20-mm radius of curvature (M2 and M3) was chosen such that a $1 / e^{2}$ waist size of $14.1 \mu \mathrm{m}$ was achieved in the crystal. Mirror M4 was mounted on a piezoelectric transducer (PZT1), used for dither-locking the OPO cavity. Mirror M2 was silver coated, allowing the pump beam to be focused directly into the crystal without using a lens. The remaining OPO cavity mirrors had a dielectric coating which transmitted the pump light and were highly reflective $(\mathrm{R}>99.9 \%)$ over the $1400-1800 \mathrm{~nm}$ region.

The OPO cavity was dither-locked to the peak of a degenerate fringe, as reported for other degenerate OPOs. A pellicle with $\mathrm{R}=28 \%$ was used to output couple $270 \mathrm{~mW}$ of signal power with $950 \mathrm{~mW}$ of incident pump power. The threshold of operation was $27 \mathrm{~mW}$, which increased to $350 \mathrm{~mW}$ when the pellicle output coupler was in place. Spectral 
broadening of this OPO (yet to be published) yields an octave spanning supercontinuum with full coverage of the 1000 $2000-\mathrm{nm}$ region, which maintains the comb spacing and offset of the original comb and is also locked to that of the Ti:sapphire comb.

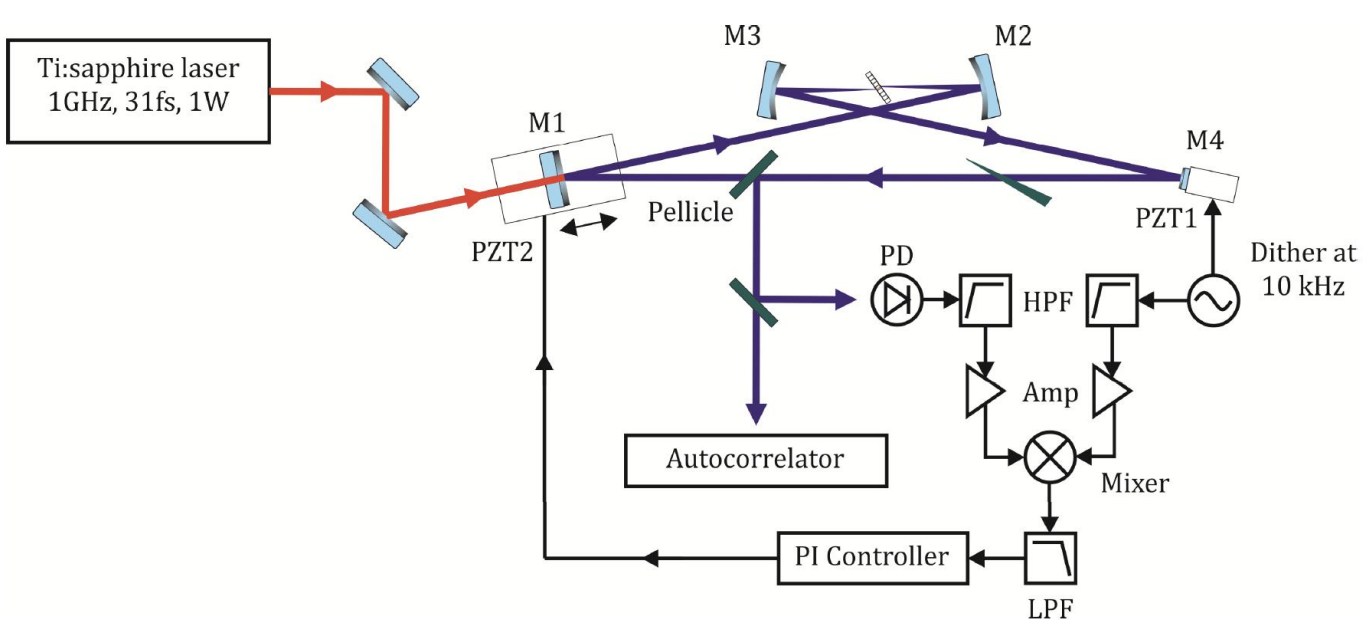

Fig. 2. Optical and electronic layout of the degenerate 1-GHz PPKTP OPO. HPF (LPF), high-pass (low-pass) filter; PD, photodiode. The OPO gain crystal is PPKTP.

\subsection{Ti:sapphire-pumped doubly-resonant degenerate 10-GHz OPOs}

The extension to direct generation of $10-\mathrm{GHz}$ pulses from a femtosecond OPO has been achieved recently, and the layout of the experiment is shown in Figure 3(a). A 10-GHz Ti:sapphire laser (Taccor x10, Laser Quantum) producing 50 -fs pulses centered at $817 \mathrm{~nm}$ with $1.4 \mathrm{~W}$ average power was used to pump the DRO. A $10-\mathrm{GHz}$ DRO of 1:1 synchronicity requires a cavity length with $\sim 30$ - $\mathrm{mm}$ optical path in a ring configuration, comprising a nonlinear crystal, a pair of 5-mm ROC mirrors and a pair of plane dielectrics, which poses acute engineering challenges. To more easily investigate the properties of a 10-GHz femtosecond DRO we selected a cavity length of $1139 \mathrm{~mm}$, corresponding to $38: 1$ synchronicity. The DRO was a 4-mirror ring cavity based on a 1-mm long Brewster-angled PPKTP crystal with $27.5 \mu \mathrm{m}$ grating period. A pair of 40-mm ROC mirrors around the crystal ensured a $1 / e^{2}$ waist of $14.0 \mu \mathrm{m}$, maintaining a 1:1 beam waist ratio with the $30-\mathrm{mm}$ long DRO cavity. A dielectric mirror acted as a pump input coupler and a broadband $10 \%$ output coupler. The pump input focusing and DRO stabilization mechanisms are identical to those reported in [22]. Approximately $1.5 \mathrm{~mm}$ of fused silica was inserted into the cavity to compensate the positive GDD of PPKTP at $1.634 \mu \mathrm{m}$ and residual GDD from the cavity mirrors.

(a)

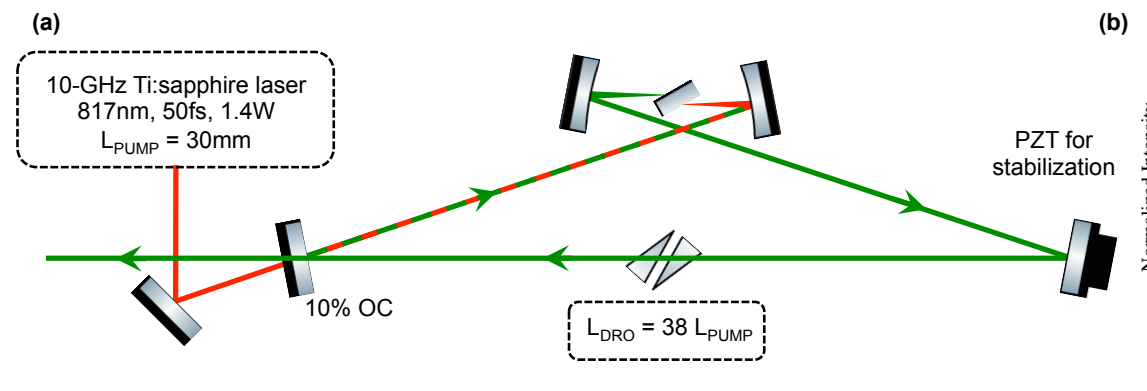

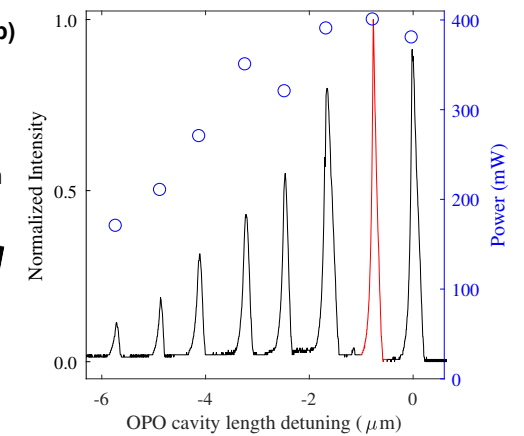

Fig. 3. (a) Schematic of the 10-GHz DRO with a cavity 38 times longer than the pump laser. (b) Oscillation peaks observed as the DRO cavity length was swept with a long-travel piezo stage. The peaks are separated by $817 \mathrm{~nm}$. The degenerate peak is indicated in red. 
Figure 3(b) shows the output power of the OPO as the cavity was swept over several microns, with corresponding output spectra shown in Figure 4(a). Only one oscillation peak was identified as degenerate (shown in red), with the remainder corresponding to partially degenerate or doubly resonant conditions. Approximately $400 \mathrm{~mW}$ of power was extracted with $1.35 \mathrm{~W}$ of incident pump power. The degenerate output spectrum had a FWHM bandwidth of 90nm, capable of supporting 46-fs pulses, and is shown in Figure 4(b). Analysis of the DRO beam profile revealed a slightly elliptical beam (87\%) despite astigmatism compensation of the Brewster-angled crystal. This is likely due to intrinsic ellipticity in the Ti:sapphire pump laser, which, along with beam divergence, becomes more prominent in compact high-repetitionfrequency cavities.

We expect that the power levels available from this system will make it possible to extend its spectral coverage using supercontinuum generation, allowing spectroscopic applications in astrocombs to be addressed.
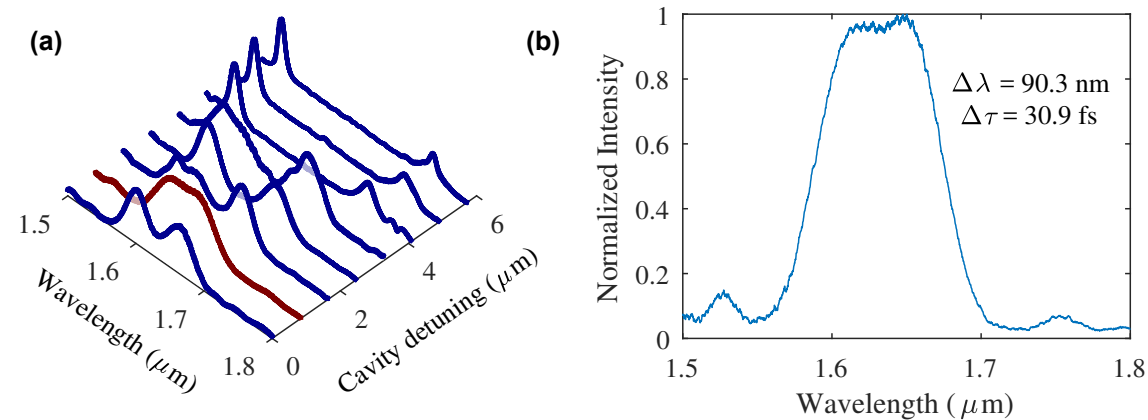

Fig. 4. (a) Discrete output spectra from the 10-GHz DRO as the cavity length is tuned over $6 \mu \mathrm{m}$.

(b) Degenerate spectrum of the DRO. The 90-nm FWHM bandwidth supports 46-fs pulses.

\subsection{Mode-resolving Fourier-transform spectroscopy}

With passively stable or fully locked frequency combs, the modes can be resolved using high-resolution spectroscopy, and the comb reconstructed from this measurement. Recently we presented a broadband Fourier-transform spectrometer [24] which is capable of sufficient resolution and bandwidth to clearly distinguish all possible filtered subsets from the previously described Fabry-Perot-filtered system, avoiding the ambiguities in determining which subset of source comb modes has been filtered. The FTS had a resolving power of $\mathrm{R}=430,000$ at $550 \mathrm{~nm}$, and was applied to the identification of comb subsets from a filtered 1-GHz supercontinuum. After apodization the FTS demonstrated an instrument line shape width of $1.26 \mathrm{GHz}$ which enabled individual comb-line positions to be identified with an uncertainty of $17.6 \mathrm{MHz}$, a relative precision of $5 \times 10^{-8}$. Correcting for air dispersion allowed the instrument to determine the comb-mode spacing to an accuracy of $300 \mathrm{~Hz}$ and filtered subsets of source comb modes to be uniquely distinguished across the entire comb bandwidth from 550 to $900 \mathrm{~nm}$. The inherently broadband design of the FTS makes it suitable in future applications for calibrating ultra-broadband astrocombs employed by instruments such as ELT HIRES. offering bandwidth from 500nm$1100 \mathrm{~nm}$. Illustrative data from the FTS are shown in Fig. 5. The input was a broadband super-continuum spectrum (Fig. 5(a)). The FTS scan was calibrated using a Rb-referenced DBR laser, giving an instrument response shown in Fig. 5(b). The comb spectrum is shown in Fig. 5(c).
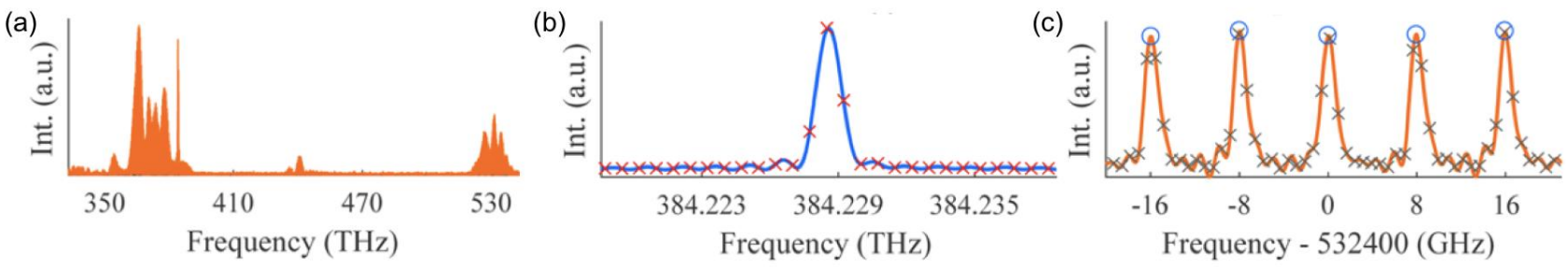

Fig. 5. (a) Input supercontinuum. (b) Unresolved Rb spectral line (1.27-GHz apodized line width) used for calibrating the FTS. (c) Retrieved supercontinuum comb modes, upsampled to illustrate the similarity of their line shapes with the instrument response. 


\section{YB:FIBER-PUMPED OPOS FOR CHEMICAL SENSING}

\subsection{Motivation}

Stand-off detection, identification of a sample at a distance without interfering with it, offers a reduced risk of harm from dangerous chemicals by avoiding the need to collect a sample for analysis, therefore eliminating the possibility of human contact with a toxic material. It has potential military applications in determining if a liquid deposition on a surface is dangerous, avoiding an unnecessary time consuming decontamination process. This is particularly important in the case of non-volatile chemical warfare agents (CWAs), as their presence persists for a long period. As an example, VX nerve agent is less volatile than water by a factor greater than 1000 [25]. A recent editorial in Optical Engineering [26] points out that, despite it being 100 years since the chemical weapons were first used on a large scale in the first world war, their use is presently an emerging threat. It argues that the easily accessible information on the internet and increased civil unrest fueling demand for improvised weapons could lead to wider use of CWAs. This scenario therefore motivates the development of stand-off detection techniques for toxic chemicals based on molecular spectroscopy. There is a range of other possible applications for such technology, including security screening and sorting of different materials e.g. plastics in a recycling context.

\subsection{Performance requirements}

While gas absorption spectroscopy is characterized by a rich spectral structure that permits species identification using carefully selected narrow absorption features, the identification of liquid, aerosol and solid samples requires a fundamentally different approach. Here, no fine spectral structure is expected, but broadband coverage is important to distinguish chemicals by examining their gross spectral shapes, which may require measurements over a few $1000 \mathrm{~cm}^{-1}$ to provide a confident diagnosis. Measurements in the mid-wave infrared (MWIR) region are useful for identifying hydrocarbon bonds, while other functional groups and their configurations can be revealed better at longer wavelengths. In particular, measurements in the long-wave infrared (LWIR) from $6-12 \mu \mathrm{m}$ can not only identify functional groups, but show differences according to their relative positions on the molecule, enabling molecular isomers to be distinguished spectroscopically.

\subsection{Yb:fiber-pumped singly-resonant MgO:PPLN OPO and application in stand-off detection of liquids}

Our original stand-off spectroscopy demonstration employed an Yb:fiber laser-pumped MgO:PPLN OPO, and is described in detail elsewhere [12]. The current embodiment of this system and its spectral coverage are shown in Fig. 6. The OPO is pumped by a chirped few-ps pulses from an $\mathrm{Yb}$ :fiber master-oscillator power amplifier. Gain is provided by a 20-mm MgO:PPLN crystal with grating periods from $27.9-31.0 \mu \mathrm{m}$, providing tuning from $2.5-4.2 \mu \mathrm{m}$.

As illustrated in Fig. 7, mid-infrared light from the OPO enters scanning Michelson interferometer in parallel with a cw reference beam used to calibrate the interferometer path length difference (Fig. 7(a)). The interferometer design is reproduced in Fig. 7(b), and example data are shown in Fig. 7(c) and (d).

Using this system we have recorded spectra using light back-scattered from non-compliant materials such as concrete. Example data are shown in Fig. 8 for the detection of a drop of the liquid TDG on a concrete surface at a distance of one meter, and for nitromethane vapor, detected by backscattered light from a concrete target at 1-m range. Extensions to this approach are possible by using sensitive detectors and larger diameter collection optics. In this way we have extended the range of this system to several tens of meters. 
CaF plane-plane mirrors

HT for $1.02-1.06 \mu \mathrm{m}, \mathrm{HT} 3-5 \mu \mathrm{m}$

HR and low GDD for 1.16 - $1.6 \mu \mathrm{m}$
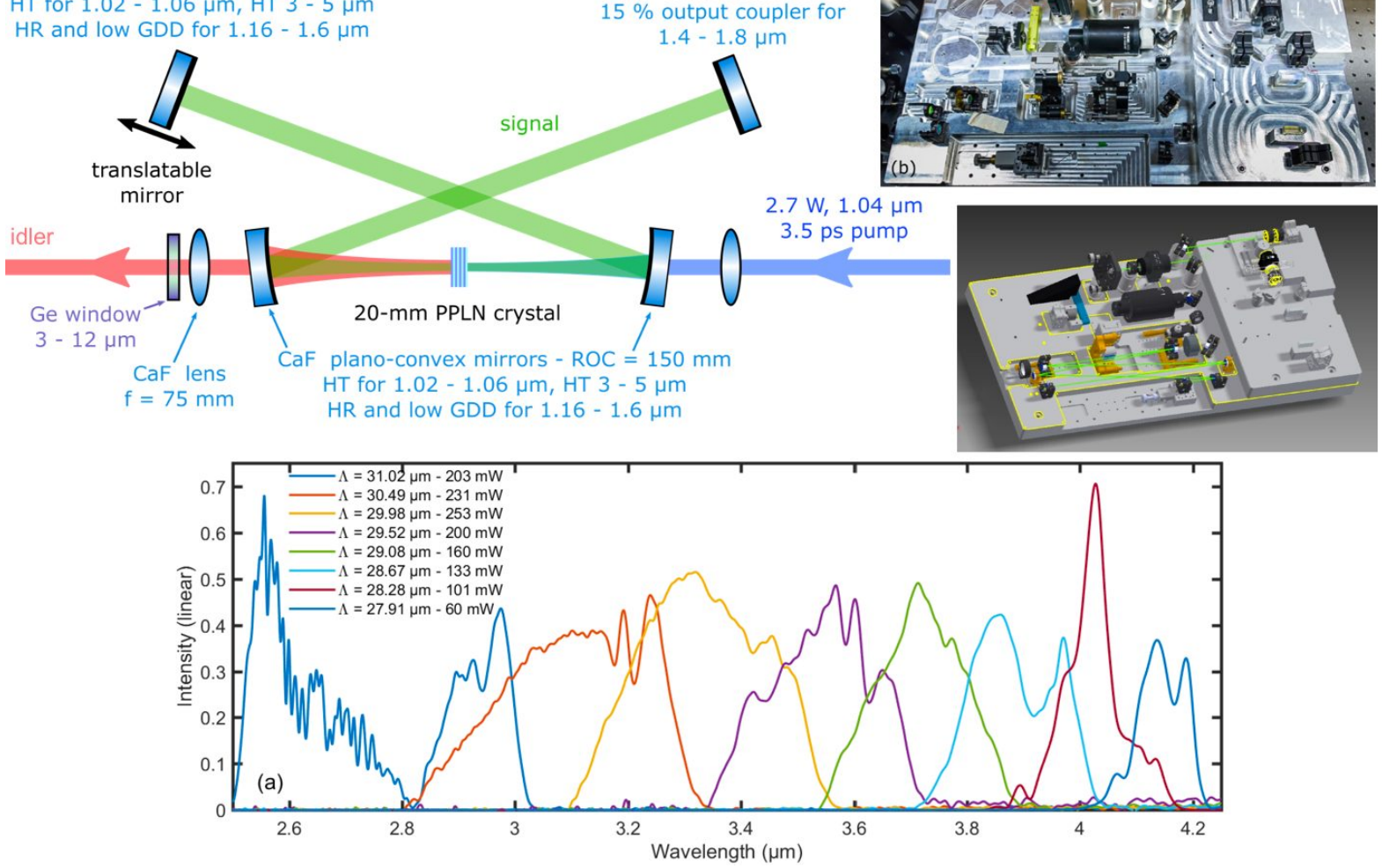

Fig. 6. MgO:PPLN OPO based on chirped-pulse pumping from an Yb:fibre laser.. The OPO idler is used for stand-off chemical sensing and tunes from $2.5-4.2 \mu \mathrm{m}$.
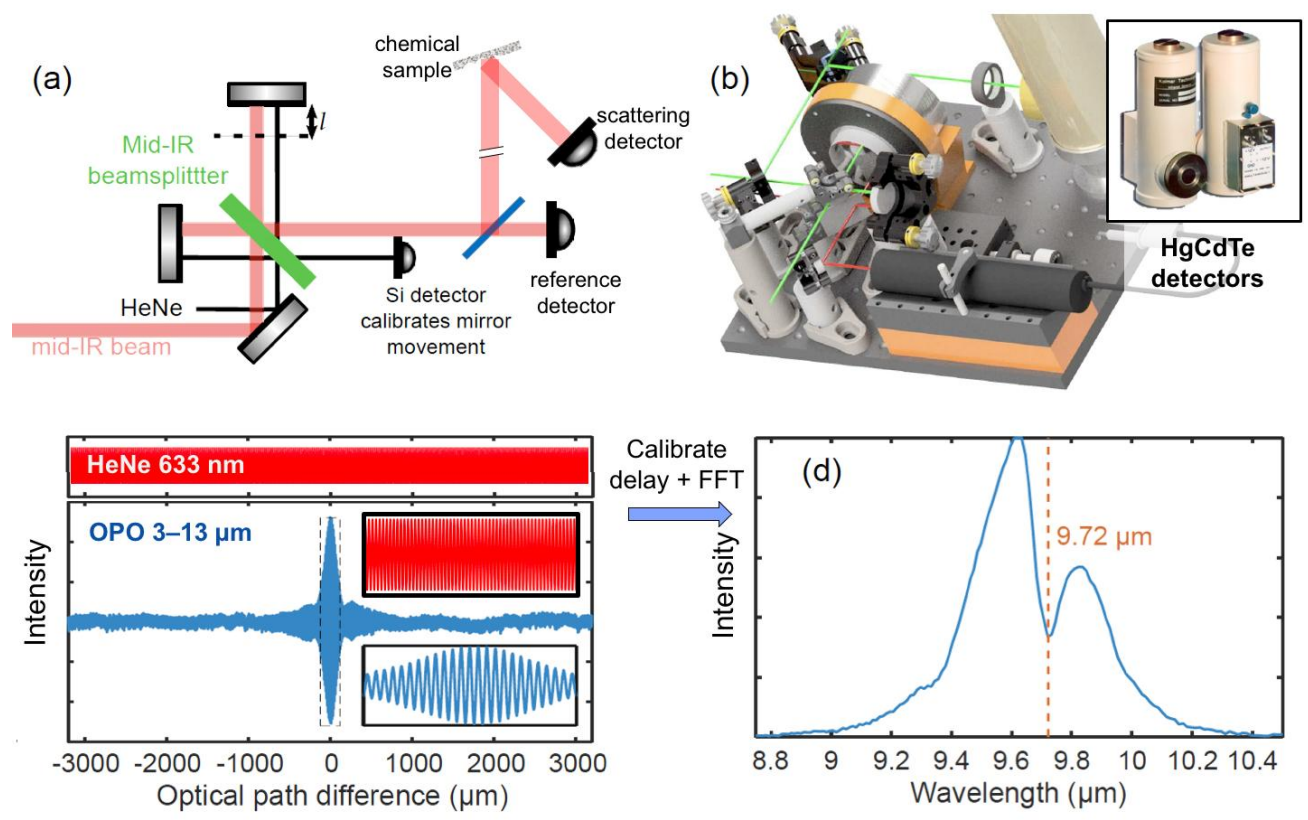

Fig. 7. Michelson interferometer and generated data. 

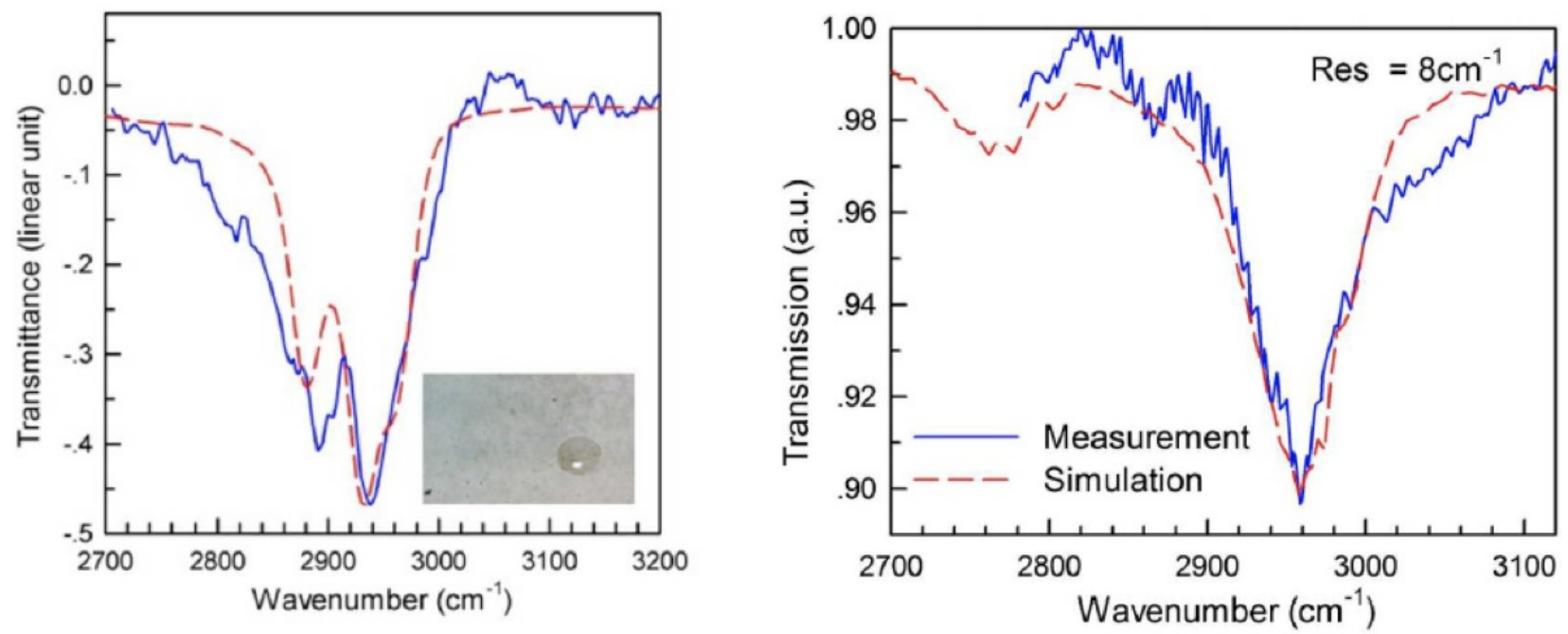

Fig. 8. Left: Measured transmission spectrum of TDG on a concrete substrate (solid lines) and comparison with reference spectrum (dashed lines). Right: Measured (solid line) and simulated (dashed line) transmission spectrum of vaporized nitromethane obtained from a concrete substrate at a stand-off distance of $1 \mathrm{~m}$.

\subsection{Yb:fiber-pumped singly-resonant OPGaP OPO and application in powder spectroscopy}

As already discussed, obtaining diagnostically useful information does not demand high resolution-liquids and solids exhibit broad absorption features, so $\mathrm{cm}^{-1}$-level resolution is sufficient - but unique identification makes it important to capture information over a broad bandwidth, making femtosecond sources ideally suited to this problem.

The preferred source for such spectroscopy was an OP-GaP femtosecond OPO $[13,14]$. The layout of the OPO is shown in Fig. 9, and it contained gratings of 21.5, 24,5, 27, 29, 31, 32.5 and $34 \mu \mathrm{m}$, individually fabricated on separate 6-mmtall coated OPGaP crystals. The beam was focused using a $40 \mathrm{~mm}$ focal length lens to a radius of $18 \mu \mathrm{m}$ in an OPGaP crystal within a signal resonant OPO ring cavity (the signal focus in the crystal is $18 \mu \mathrm{m}$ ). The coating of one spherical mirror was optimized for transmission at the pump wavelength of $1040 \mathrm{~nm}$ and for high reflectivity at the resonant signal wavelength in a range from $1.15-1.35 \mu \mathrm{m}$, while the other spherical mirror collimated the idler beam emerging from the OPGaP crystal and was silver coated to provide high reflectivity for all idler wavelengths. Using a metal mirror rather than a lens to collimate the idler beam also avoids chromatic aberration issues when tuning the wavelength.

For powder spectrocopy the beam was directed through a scanning Michelson interferometer (acting as a Fourier transform spectrometer) before being directed vertically onto a white powder sample ( $\sim 10 \mathrm{~g}$ in mass $)$ on a card. The illumination was normal to the card, with diffuse light scattered over the full range of solid angles. To avoid spectroscopic contributions from the substrate we chose to collect light scattered at nearly $90^{\circ}$ to the incident beam and focused this onto a mercury cadmium telluride detector (Fig. 10(a)). The spectrum of the light scattered from caffeine powder was measured by Fourier transforming the interference fringes detected, using three different OPO gratings $(\Lambda=$ 29,31 and $32.5 \mu \mathrm{m}$ ) to generate broadband incident radiation from 8-11 $\mu \mathrm{m}$. The illumination and scattered spectra are shown in Fig. 10(b), each one based on an average of 7 measurements recorded in $250 \mathrm{~ms}$ at $2 \mathrm{~cm}^{-1}$ resolution. The scattered spectrum in each case is divided by the illumination spectrum to find the spectral response of the powder, shown in Fig. 10(c-e), revealing clear absorption features.

A library of powder scattering spectra was compiled by collecting 14 interferograms of 11 white powder samples including caffeine (the samples are listed in Table 1) using the 29 and $31 \mu \mathrm{m}$ gratings. The powders were chosen as they are readily available and require minimal safety precautions when handling. Examples of the library spectra between wavelengths of 8.2-8.9 $\mu \mathrm{m}$, recorded using the $29 \mu \mathrm{m}$ grating are shown in Fig. 11. To compare a spectrum with the library, Pearson's correlation coefficient was used, which is particularly suitable as it is insensitive to differences in magnitude between compared signals, since it only recognizes the shape and position of features, and returns a maximum 
value of 1 for perfect correlation. To classify a spectrum, its correlation coefficient with respect to every library spectrum was found, and the correlation values for each particular chemical were averaged, giving a single correlation value for each powder. The library match was then considered to be the chemical with the highest coefficient. This approach worked perfectly to classify any individual spectrum recorded at the same time as the library data was recorded.
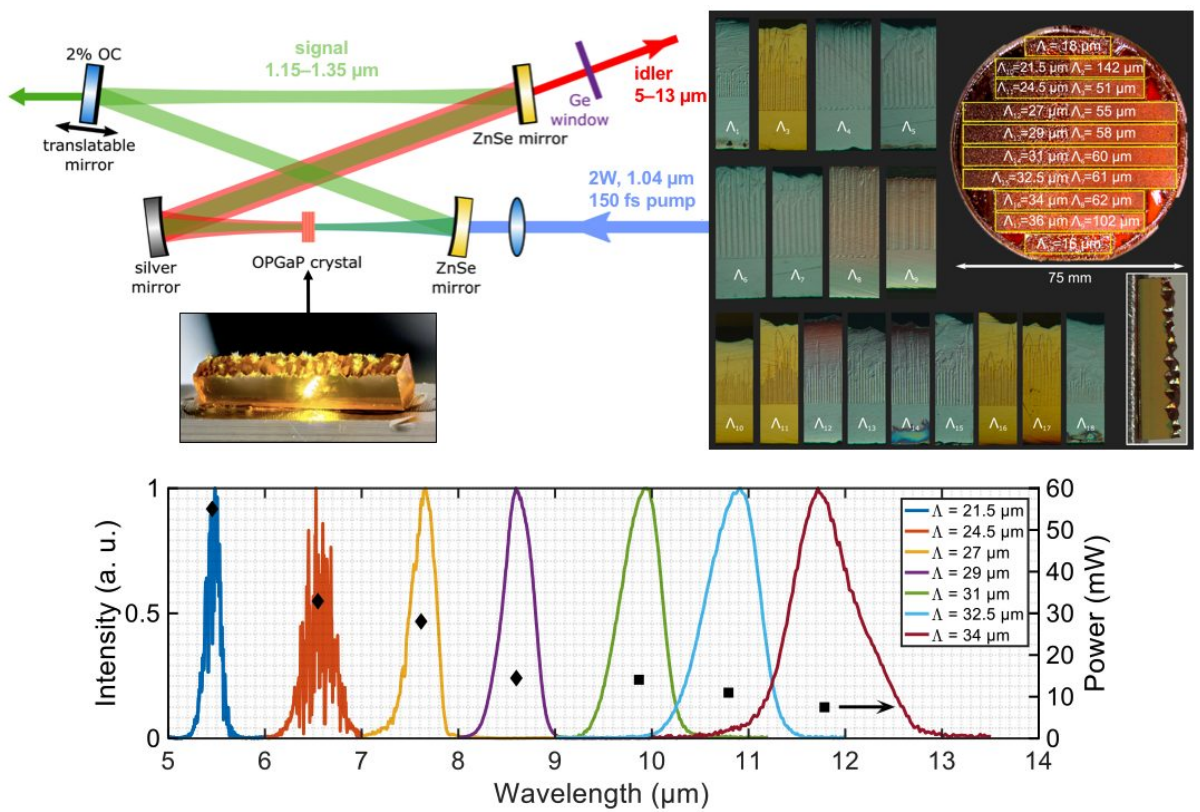

Fig. 9. OPGaP OPO (top left) fabricated using crystals diced from a single OPGaP wafer (top right), and tuning achieved on the different gratings.
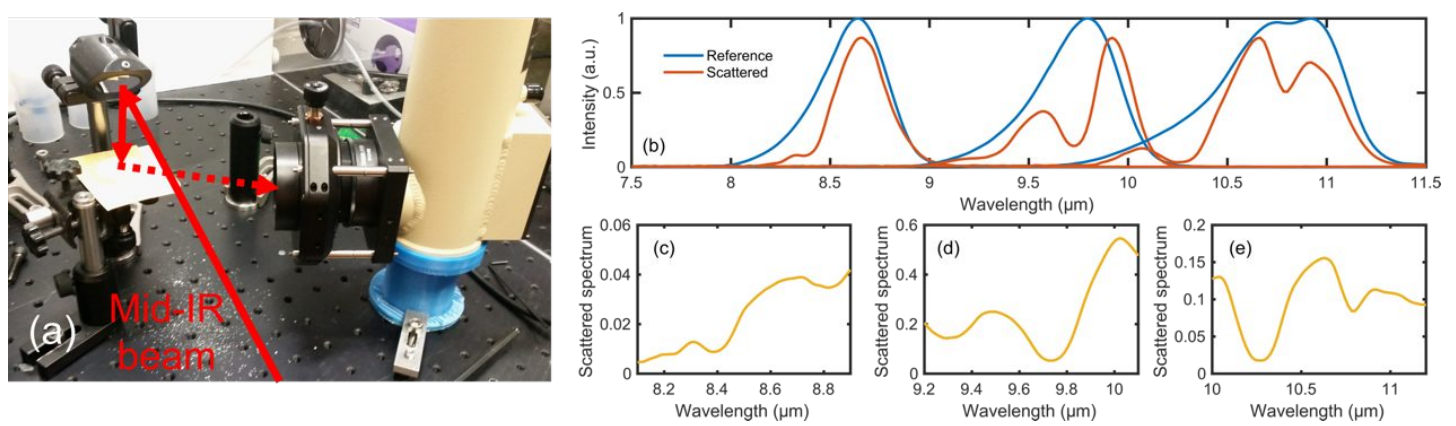

Fig. 10. (a) Mid-IR beam path, showing the detector collecting the scattered light. (b) Spectra from 3 different OPO gratings (blue) and spectra of light scattered by caffeine powder (orange). (c)-(e) Left to right: spectral response of caffeine for grating periods of 29,31 and $32.5 \mu \mathrm{m}$.
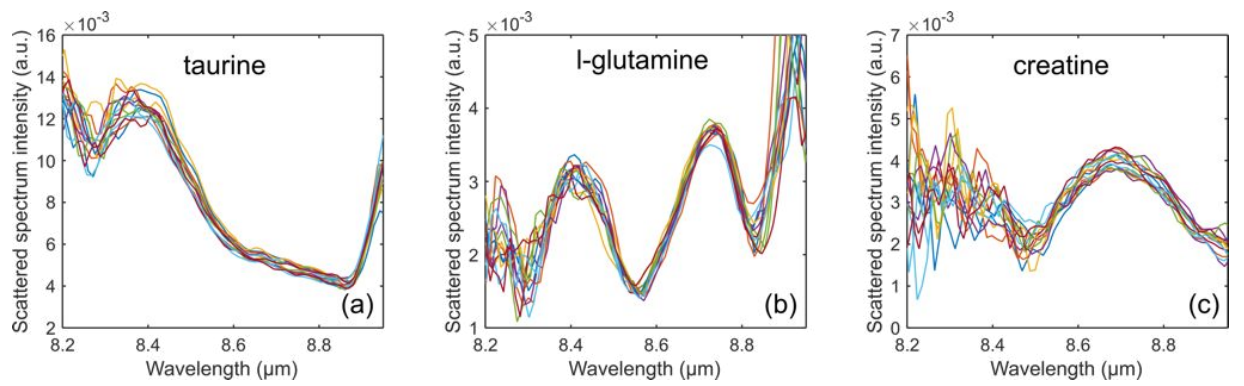

Fig. 11. Library scattered spectra for powders of (a) taurine, (b) l-glutamine and (c) creatine. 
To test the robustness of the library classification scheme, new data were recorded at a later time, after reconstructing the scattering setup and using new samples of the same powders. The signal strengths of these data were weaker than the library data. Table 1 presents representative results, displaying the mean correlation coefficient of three tests with each library chemical. As indicated by colored cells, taurine and l-glutamine were matched correctly, while creatine was not and matched equally with the taurine and creatine library data.

Table 1. Mean correlation coefficient of three test powders with each library powder.

\begin{tabular}{|c|c|c|c|c|c|c|c|c|c|c|c|}
\hline \multirow[b]{2}{*}{ Test powders } & \multicolumn{11}{|c|}{ Library } \\
\hline & caffeine & aspirin & paracetamol & $\mathrm{n}$ acetyl I cysteine & I-glutamine & inistol & taurine & leucine & creatine & beta analine & dextrose \\
\hline taurine & -0.96 & -0.70 & -0.89 & -0.72 & -0.44 & -0.48 & 0.95 & 0.34 & -0.06 & -0.52 & -0.72 \\
\hline I-glutamine & 0.53 & 0.51 & 0.52 & 0.69 & 0.89 & 0.47 & -0.36 & -0.21 & -0.26 & 0.61 & 0.34 \\
\hline creatine & -0.57 & -0.63 & -0.40 & -0.70 & -0.45 & -0.20 & 0.53 & 0.36 & 0.53 & -0.22 & -0.09 \\
\hline
\end{tabular}

The library classification scheme definitively identified spectra from powders with distinctive spectral features between $8.2-8.9 \mu \mathrm{m}$, even when measurements were made again with weaker signals. Fig. 11(a) and (b) show the distinctive spectra of taurine and l-glutamine which were easily matched. (c) shows the library spectra for creatine, which is weaker and noisier, with less distinctive features. This is the reason that creatine scattering measured later was not clearly identified by the library.

By extending the library across a greater wavelength range, it would be possible to correctly identify a wider range of powders with greater confidence.

\subsection{Yb:fiber-pumped dual combs based on MgO:PPLN and OPGaP OPOs}

Dual-comb spectroscopy [5] is a laser-based analog to Fourier-transform spectroscopy, enabling rapid, high-resolution measurements in a system containing no moving parts. Using dual MgO:PPLN and OPGaP OPOs, we have demonstrated dual-comb spectroscopy in the 3- to 4- $\mu \mathrm{m}$ and 6- to $8-\mu \mathrm{m}$ regions. The general system architecture is shown in Fig. 12.

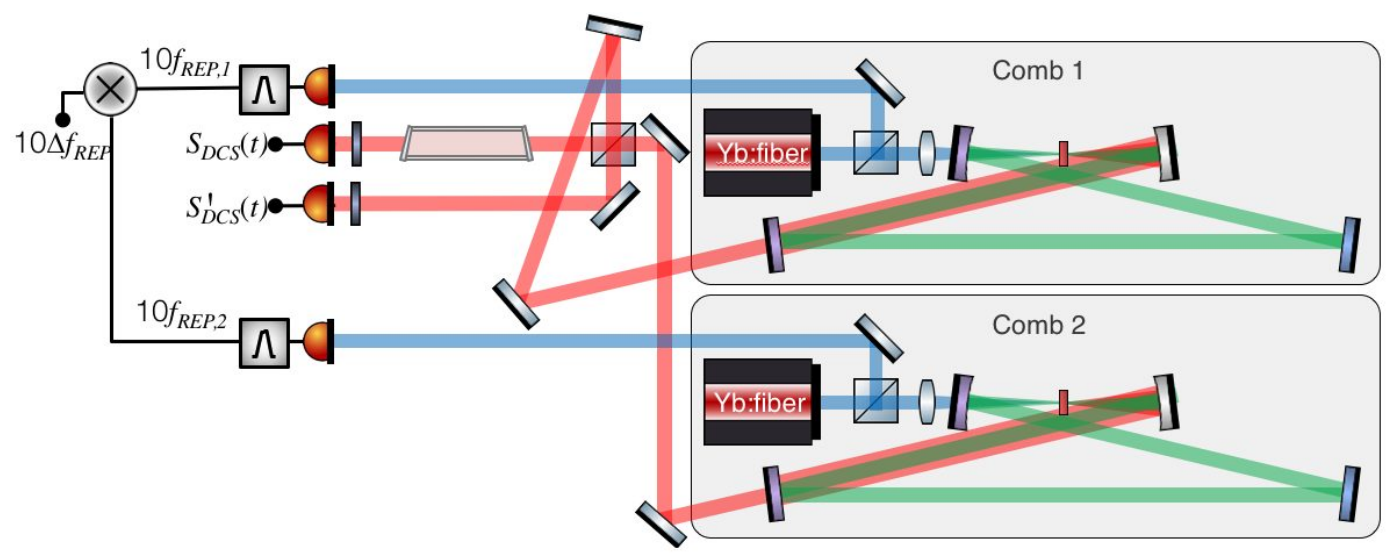

Fig. 12. Dual Yb:fibre master-oscillator power amplifiers are used to pump two OPOs containing either MgO:PPLN or OPGaP crystals as their gain media. Mid-IR idler light is out-coupled from the cavity after collection using a silver mirror. Output idler pulses from the two OPOs are combined before and after passing through a gas cell, then detected individually on fast MCT detectors. Two silicon photodiodes record the pulse repetition frequencies for calibration purposes. The pump pulse durations are approximately $200 \mathrm{fs}$.

\subsubsection{Dual-comb spectroscopy using MgO:PPLN OPOs}

Two identical $\mathrm{Yb}$ :fiber oscillators with slightly different repetition rates were used to seed corresponding $\mathrm{Yb}$ :fiber amplifiers, producing approximately 3-ps chirped pulses from each channel with average powers of $2.3 \mathrm{~W}$, center wavelengths of $1055 \mathrm{~nm}$ and bandwidths $>20 \mathrm{~nm}$. The repetition rate $\left(f_{\text {REP }}\right)$ of each pump was $102 \mathrm{MHz}$, while the repetition-frequency difference $\left(\triangle f_{R E P}\right)$ between the two channels was free-running at $496 \mathrm{~Hz}$. The seed oscillators shared 
a single breadboard to provide common-mode rejection of environmental fluctuations. Each OPO contained a 20-mmlong $5 \mathrm{~mol} . \% \mathrm{MgO}: \mathrm{PPLN}$ crystal with a grating period of $30.5 \mu \mathrm{m}$. In this scheme, the pump bandwidth and spectral shape efficiently are transferred to the idler pulses [27]. The two identical idler pulses were combined on a $\mathrm{CaF}_{2}$ beamsplitter before entering a $20-\mathrm{cm}$ cell, containing a $1 \mathrm{~atm}$ mixture of $\mathrm{N}_{2}$ with a low concentration $(<1 \%)$ of $\mathrm{CH}_{4}$. The DC interferogram signal was detected by two cryogenically cooled MCZT ( $\mathrm{HgCdZnTe}$ ) balanced detectors with a bandwidth of $100 \mathrm{MHz}$. After Nyquist filtering to $<50 \mathrm{MHz}$, the DC interferogram was sampled at 12-bit resolution at a rate of $200 \mathrm{MHz}$. The signals $f_{R E P}, 10 \triangle f_{R E P},\left(=f_{R E P I}-f_{R E P 2}\right), f_{C E O I}$ and $f_{C E O 2}$ [6] were co-recorded with the DC interferogram.

Wavelength calibration proceeded by first making a simple comparison of the mode frequencies and the carrier frequency of the DC interferogram to identify the CEO frequencies to be either correct as measured or to be $f_{R E P}-f_{C E O}$. Following this, a wavelength range from $3.2-3.4 \mu \mathrm{m}$ was defined (corresponding to mode numbers from 864437 918464) and the comb-mode frequencies $f_{1}$ and $f_{2}$ calculated to create a look-up table which uniquely mapped the DC carrier frequency to the corresponding wavelength, $\lambda=c\left(f_{1}+f_{2}\right) / 2 f_{1} f_{2}$.

The instantaneous frequencies of the $10 \triangle f_{R E P}, f_{C E O 1}$ and $f_{C E O 2}$ signals were obtained directly in the time domain using a zero-crossings algorithm to produce an average for each frequency within the time window corresponding to each individual interferogram $(\sim 100 \mu \mathrm{s})$. After Fourier transforming, these frequencies were used to perform the calibration procedure independently on each spectrum. Full details of this procedure appear in [28]. The result is shown in Fig. 13. After calibration the spectra exhibited a standard deviation in wavelength of $2.1 \mathrm{~nm}\left(317 \mathrm{mHz}\right.$ in $\left.\Delta f_{R E P}\right)$. By automatically re-centering the data around the median wavelength value it was possible to align all the recorded spectra with sub-nm precision and average them, improving the signal:noise ratio by $\times 5$ and achieving close correspondence with the Hitran absorbance simulation after background subtraction (Fig. 13(b)).

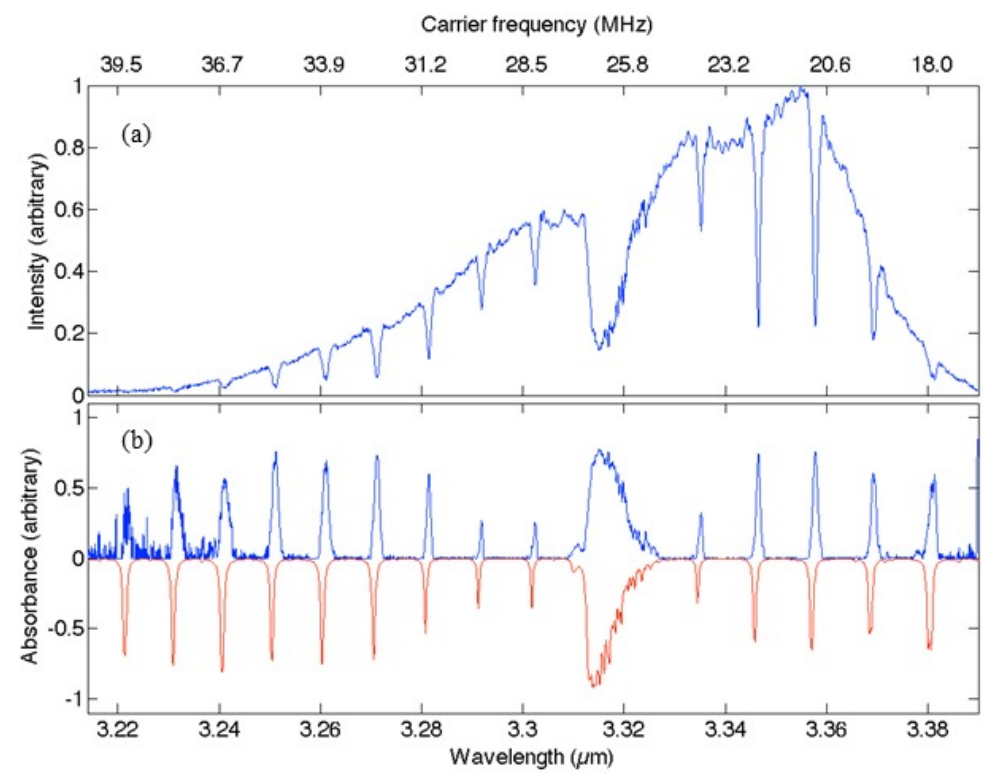

Fig. 13. (a) Average of 23 calibrated spectra after re-centering on the median wavelength, and (b) a comparison of the calculated absorbance (blue) with the equivalent Hitran data (red).

\subsubsection{OPGaP OPOs based dual-comb spectrometer}

The OPGaP DCS system comprised two entirely free-running OPGaP OPOs pumped by two identical femtosecond $\mathrm{Yb}$ :fiber lasers whose pulse repetition frequencies (nominally $102.0 \mathrm{MHz}$ ) could be adjusted using an intracavity piezoelectric actuator. The OPGaP OPOs were operated on grating periods of $24.5 \mu \mathrm{m}$ to obtain signal (idler) wavelengths centered around $1.25 \mu \mathrm{m}(6.5 \mu \mathrm{m})$ and $27 \mu \mathrm{m}$ for signal (idler) wavelengths centered near $1.22 \mu \mathrm{m}$ $(7.5 \mu \mathrm{m})$. The instantaneous bandwidth of the idler pulses in each case was approximately $500 \mathrm{~nm}$. Dual-comb interferograms were measured from both channels of the beamsplitter using $\mathrm{LN}_{2}$-cooled $\mathrm{HgCdTe}$ detectors. Up to 120 interferograms were recorded in a single acquisition, following which each interferogram was automatically centered and 
Fourier-transformed to yield its radio-frequency spectrum. Simultaneously the $10^{\text {th }}$ harmonic of $\Delta f_{R E P}$ was recorded directly in the time domain to provide an average $\Delta f_{R E P}$ value for each individual interferogram, after which the corresponding RF spectrum was scaled to the optical domain by multiplying its frequency sampling interval by a factor of $f_{R E P} / \Delta f_{R E P}$. By using a full-spectrum cross-correlation technique, each spectrum was accurately co-aligned with every other spectrum $[7,28]$. In this way, the systematic frequency shifts between all the members of a dataset containing more than one hundred consecutively acquired spectra were corrected to provide a single high-quality spectrum.

The results of this procedure are shown in Fig. 14 for operation of the OPOs in the $6.3-6.7-\mu \mathrm{m}$ wavelength region $\left(1500-1590 \mathrm{~cm}^{-1}\right)$ and the $7.3-7.8-\mu \mathrm{m}$ region $\left(1280-1370 \mathrm{~cm}^{-1}\right)$. Full details appear in [7].

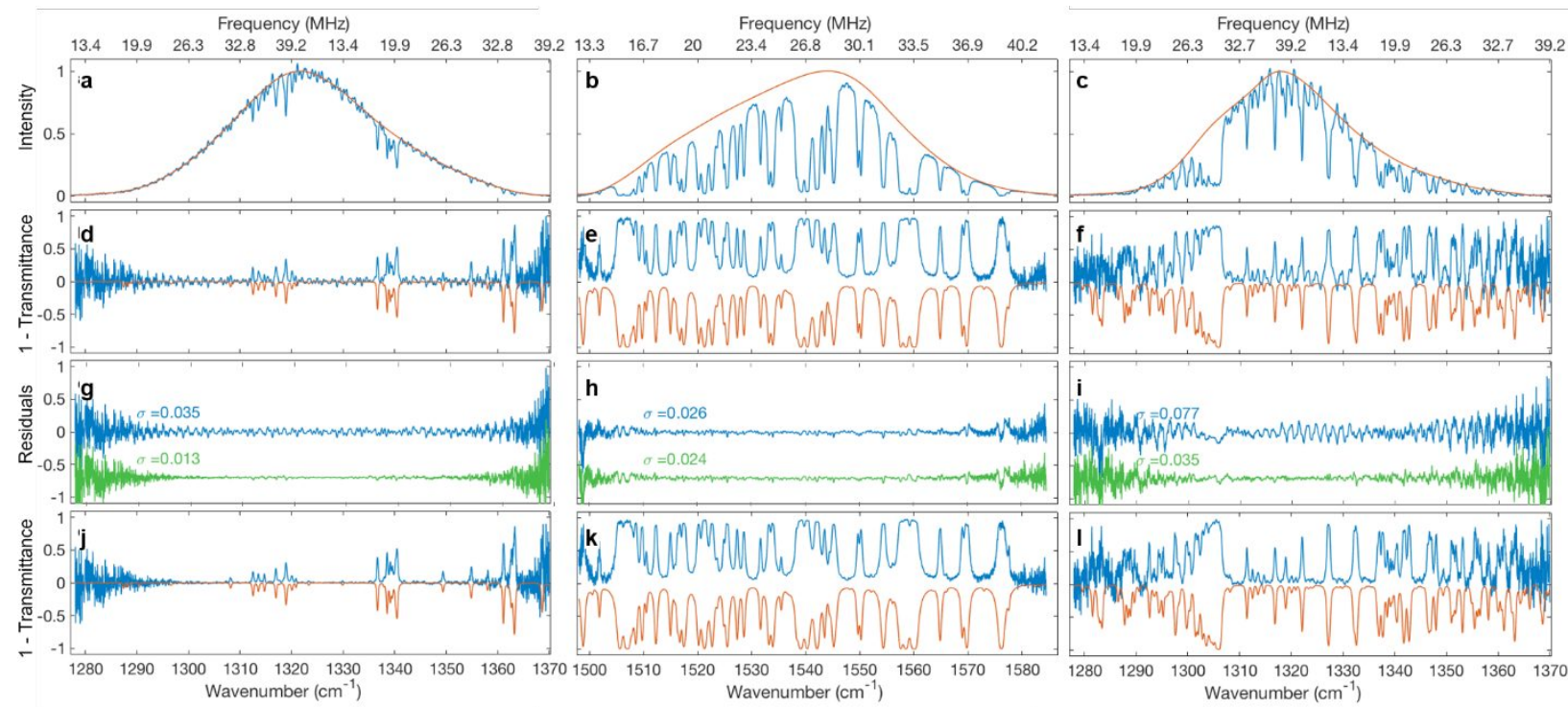

Fig. 14. Results of dual-comb fingerprint-region spectroscopy of ambient air (left and center) and with the additional of a $20-\mathrm{cm}$ cell containing a nominal $2 \%$ methane in air mix (right). a, b, c. Averaged dual-comb spectra (blue) and fitted envelope (red); d, e, f. Extracted transmittance from the dual-comb data and comparison with a HITRAN 2012 fit, from which the spectroscopy resolution and gas concentrations are obtained. g, h, i. Residuals of the original fit (blue) and after étalon removal (green, vertically displaced by -0.6 for clarity). j, $\mathrm{k}, 1$. Transmittances after étalon removal, showing excellent agreement with the HITRAN 2012 lineshapes, magnitudes and positions.

\section{CONCLUSIONS}

We have reviewed here a number of OPO configurations and their embodiments in several novel spectroscopic systems. Developments of these techniques are expected to allow such OPOs to be adopted in spectrometers ranging from those within large telescopes to field-based $\sim 100$-m range standoff spectroscopy systems.

\section{REFERENCES}

[1] Giordmaine, J. A. and Miller, R. C., "Tunable Coherent Parametric Oscillation in LiNbO3 at Optical Frequencies," Phys. Rev. Lett. 14(24) 973-976 (1965).

[2] Edelstein, D. C., Wachman, E. S. and Tang, C. L., "Broadly tunable high repetition rate femtosecond optical parametric oscillator," Appl. Phys. Lett. 54(18), 1728-1730 (1989).

[3] Burr, K.C. and Tang, C. L., "Femtosecond midinfrared-induced luminescence study of the ultrafast dynamics of split-off holes in GaAs," Appl. Phys. Lett. 74(12), 1734-1736 (1999).

[4] Tillman, K. A., Maier, R. R. J., Reid, D. T. and McNaghten, E. D., "Mid-infrared absorption spectroscopy across a $14.4 \mathrm{THz}$ spectral range using a broadband femtosecond optical parametric oscillator," Appl. Phys. Lett. 85(16), 3366-3368 (2004).

[5] Coddington, I., Newbury, N. and Swann, W., "Dual-comb spectroscopy," Optica 3(4), 414-426 (2016). 
[6] Zhang, Z. W., Gardiner, T, Reid, D. T., "Mid-infrared dual-comb spectroscopy with an optical parametric oscillator," Optics Letters 38(16), 3148-3150 (2013).

[7] Kara, O, Maidment, L, Gardiner, T, Schunemann, P. G., Reid, D. T., "Dual-comb spectroscopy in the spectral fingerprint region using OPGaP optical parametric oscillators," Optics Express 25(26), 32713-32721 (2017).

[8] Balskus, K, Schilt, S, Wittwer, V. J., Brochard, P, Ploetzing, T, Jornod, N, McCracken, R. A., Zhang, Z, Bartels, A, Reid, D. T., Sudmeyer, T, "Frequency comb metrology with an optical parametric oscillator," Optics Express 24(8), 8370-8381 (2016).

[9] Steinmetz, T., Wilken, T., Araujo-Hauck, C., Holzwarth, R., Hänsch, T. W., Pasquini, L., Manescau, A, D'Odorico, S, Murphy, M. T., Kentischer, T, Schmidt, W, Udem, T, "Laser frequency combs for astronomical observations," Science 321(5894), 1335-1337 (2008).

[10] McCracken, R. A., Charsley, J. M., Reid, D. T., "A decade of astrocombs: recent advances in frequency combs for astronomy," Optics Express 25(13), 15058-15078 (2017).

[11]Marconi, A, Di Marcantonio, P, D'Odorico, V, Cristiani, S, Maiolino, R, Oliva, E, Origlia, L, Riva, M, Valenziano, L, Zerbi, F. M., Abreu, M, Adibekyan, V, Prieto, C. A., Amado, P. J., Benz, W, Boisse, I, Bonfils, X, Bouchy, F, Buchhave, L, Buscher, D, Cabral, A, Martins, B. L. C., Chiavassa, A, Coelho, J, Christensen, L. B., Delgado-Mena, E, De Medeiros, J. R., Di Varano, I, Figueirall, P, Fisher, M, Fynbo, J. P. U., Glasse, A. C. H., Haehnelt, M, Haniff, C, Hansen, C. J., Hatzes, A, Huke, P, Korn, A. J., Leao, IC, Liske, J, Lovis, C, Maslowski, P, Matute, I, McCracken, R. A., A. P., Monteiro, M. J. P. F. G., Morris, S, Morris, T, Nicklas, H, Niedzielski, A, Nunes, N. J., Palle, E, Parr-Burman, P, Parro, V, Parry, I, Pepe, F, Piskunov, N, Queloz, D, Quirrenbach, A, Lopez, R. R., Reiners, A, Reid, D. T., Santos, N, Seifert, W, Sousa, S, Stempels, H. C., Strassmeier, K, Sun, X, Udry, S, Vanzi, L, Vestergaard, M, Weber, M, Zackrisson, E, "EELT-HIRES the highresolution spectrograph for the E-ELT," Ground-Based and Airborne Instrumentation for Astronomy VI, Proceedings of SPIE 9908, UNSP 990823 (2016).

[12]Zhang, Z. W., Clewes, RJ, Howle, C. R., Reid, D. T., "Active FTIR-based stand-off spectroscopy using a femtosecond optical parametric oscillator," Optics Letters 39(20), 6005-6008 (2014).

[13] Maidment, L, Schunemann, P. G., Reid, D. T., "Molecular spectroscopy from 5-12 $\mu \mathrm{m}$ using an OP-GaP OPO," Nonlinear Frequency Generation and Conversion: Materials and Devices XVI, Proceedings of SPIE 10088, UNSP 100880Y (2017).

[14] Maidment, L, Schunemann, P. G., Reid, D. T., "Molecular fingerprint-region spectroscopy from 5 to $12 \mu \mathrm{m}$ using an orientation-patterned gallium phosphide optical parametric oscillator," Optics Letters 41(18), 42614264 (2016).

[15] Murphy, M. T., Udem, T., Holzwarth, R., Sizmann, A., Pasquini, L., Araujo-Hauck, C., Dekker, H., D’Odorico, S., Fischer, M., Hänsch, T., and Manescaur, A.,"High-precision wavelength calibration of astronomical spectrographs with laser frequency combs," Mon. Not. R. Aston. Soc. 380, 839-847 (2007).

[16] http://www.exoplanet.eu. Accessed: 7 May 2017.

[17]Fischer, DA, Anglada-Escude, G, Arriagada, P, Baluev, RV, Bean, JL, Bouchy, F, Buchhave, LA, Carroll, T, Chakraborty, A, Crepp, JR, Dawson, RI, Diddams, SA, Dumusque, X, Eastman, JD, Endl, M, Figueira, P, Ford, EB, Foreman-Mackey, D, Fournier, P, Furesz, G, Gaudi, BS, Gregory, PC, Grundahl, F, Hatzes, AP, Hebrard, G, Herrero, E, Hogg, DW, Howard, AW, Johnson, JA, Jorden, P, Jurgenson, CA, Latham, DW, Laughlin, G, Loredo, TJ, Lovis, C, Mahadevan, S, McCracken, TM, Pepe, F, Perez, M, Phillips, DF, Plavchan, PP, Prato, L, Quirrenbach, A, Reiners, A, Robertson, P, Santos, NC, Sawyer, D, Segransan, D, Sozzetti, A, Steinmetz, T, Szentgyorgyi, A, Udry, S, Valenti, JA, Wang, SX, Wittenmyer, RA, Wright, JT, "State of the Field: Extreme Precision Radial Velocities," Publications of the Astronomical Society of the Pacific 128(964), 66001 (2016).

[18] Marcy, G. W. and Butler, R. P., "Precision radial velocities with an iodine absorption cell," Publications of the Astronomical Society of the Pacific 104, 270 (1992).

[19]Butler, R. P., Marcy, G. W., Williams, E., McCarthy, C., Dosanjh, P. and Vogt, S. S., "Attaining Doppler Precision of $3 \mathrm{~m} \mathrm{~s}-1$," PASP 108, 500 (1996).

[20]Mayor, M. and Queloz, D., "A Jupiter-mass companion to a solar-type star". Nature. 378 (6555), 355-359 (1995).

[21]Huke, P., Origlia, L., Riva, M., Pepe, F., Reiners, A., Charsley, J. M., McCracken, R. A., Reid, D. T., Kowzan, G., Maslowski, P., Korhonen, H., Broeg, C., Dolon, F., Boisse, I., Disseau, K., Perruchot, S., Ottogalli, S., and Bandy, T., "Phase A: Calibration concepts for HIRES," in [Optical Measurement Systems for Industrial Inspection X], Proc. SPIE , $10329\{91$ (2017). 
[22]McCracken, R. A., Depagne, E, Kuhn, R. B., Erasmus, N, Crause, L. A., Reid, D. T., "Wavelength calibration of a high resolution spectrograph with a partially stabilized $15-\mathrm{GHz}$ astrocomb from 550 to $890 \mathrm{~nm}$," Optics Express 25(6), 6450-6460 (2017).

[23] McCracken, R. A., Reid, D. T., "Few-cycle near-infrared pulses from a degenerate $1 \mathrm{GHz}$ optical parametric oscillator," Optics Letters 40(17), 4102-4105 (2015).

[24]Charsley, J. M., McCracken, R. A., Reid, L, Reid, D. T., "Broadband Fourier-transform spectrometer enabling modal subset identification in Fabry-Perot-based astrocombs," Optics Express 25(16), 19251-19261 (2017).

[25]Meyer, E., "Standoff Detection of Persistent Chemical Agents on Surfaces, Defense Threat," Reduction Agency Report 298 (2010).

[26]Fountain, A. W. , "Chemical, Biological, Radiological, and Explosive Sensing, Optical Engineering," 53, 021101 (2014).

[27]Zhang, Z. W., Gu, C. L., Sun, J. H., Wang, C. Y., Gardiner, T, Reid, D. T., "Asynchronous midinfrared ultrafast optical parametric oscillator for dual-comb spectroscopy," Optics Letters 37(2), 187-189 (2012).

[28]Kara, O, Zhang, Z, Gardiner, T, Reid, D. T., "Dual-comb mid-infrared spectroscopy with free-running oscillators and absolute optical calibration from a radio-frequency reference," Optics Express 25(14), 1607216082 (2017). 